\title{
Preparation Methods and Anti-hepatoma Effects of Difunctional Hyaluronic Acid Nano-micelle
}

\author{
Guixiang Tian, Bo Zhang, Bo Lian, Xiaohui Qi, Zhiqin Gao, Jingliang Wu* \\ School of Bioscience and Technology, Weifang Medical University, Weifang, Shandong Province, China \\ *Corresponding author
}

Keywords: doxorubicin; hyaluronic acid; nano-micelle; folic acid

\begin{abstract}
Difunctional nano-micelle is prepared from hyaluronic acid modified with folic acid (FA-HA) and hyaluronic acid modified with histidine (His-HA). Doxorubicin (DOX) is selected as the drug to prepare drug loaded nano-micelle; the particle size and Zeta potential of nano-micelle are analyzed. The inhibition rate of nano-micelle on the growth of human hepatoma cells (HepG2) is detected by MTT method; the anti-tumor activity of nano-micelle is analyzed in vivo. The results show that drug loading mixed micelles are spherical with the particle size of 238.4-312.7 nm. The degree of dispersion is good, and the Zeta potential is $-12.5 \sim-9.8 \mathrm{~m} \mathrm{~V}$. The anti-tumor experiment in vitro and in vivo show that the drug loaded nano-micelle is more effective in anti-hepatoma than doxorubicin.
\end{abstract}

\section{Introduction}

Liver cancer (hepatoma) is one of the malignant tumors which threaten the life and health of human beings. Surgery and chemotherapy are the main means of treatment at present. The great side effects of chemotherapy cause the tumor to relapse and transfer easily ${ }^{[1-2]}$. The precise targeted therapy has become the focus of antitumor research ${ }^{[3-4]}$.

Doxorubicin can inhibit the synthesis of DNA and RNA. It is a periodic nonspecific drug, and has killing effects on many kinds of tumors. However, it has many side effects, such as cardiac toxicity and nephrotoxicity. So it cannot be widely applied in clinical tumor chemotherapy ${ }^{[5]}$. The nano drug delivery system can realize the precise delivery of doxorubicin, which can improve the bioavailability of doxorubicin and reduce its toxicity and side effects ${ }^{[6-7]}$.

HA is a linear, negatively charged natural polysaccharide. It includes two alternate units: D-glucuronic acid and N-acetyl -D- glucosamine. It is a biocompatible, biodegradable polysaccharide which widely exists in extracellular tissue fluid. CD44 receptor has high expression in various cancer cells, while HA can specifically bind to CD44 receptor. HA as a drug delivery carrier is extensively studied in the field of drug delivery ${ }^{[8-10]}$.

On the basis of previous studies, researchers design a kind of difunctional nano-micelle with tumor targeting and $\mathrm{pH}$ responsiveness. Hyaluronic acid (HA) is used as carrier material. His-HA and FA-HA polymers are synthesized through histidine (His) and folic acid as modifiers. DOX hyaluronic acid mixed nano-micelles are prepared, and their physicochemical properties and anti-hepatoma activity in vivo and in vitro are analyzed.

\section{Research Material and Method}

\subsection{Experimental cells and animals}

HepG2 cells are purchased from the life collection center of Wuhan University; H22 cells are given by the Key Laboratory of Applied Pharmacology of Weifang Medical University. SPF grade (clean grade) Kunming mice are purchased from the experimental animal center of Weifang Medical University. These male mice are 6-8 weeks old, weighing $(20+2)$ g. The number of mice is SCXK (Lu) 20160007. 


\subsection{Main instruments and reagents}

Hyaluronic acid (HA) is purchased from Shandong Freda Biological Pharmaceutical Co., Ltd.; histidine (His) and folic acid (FA) are purchased from Sigma company; triazine condensation agent (DMT-MM) is purchased from Shanghai Gongjia Biological Technology Co. Ltd.; doxorubicin (DOX-HCl) is purchased from Shanxi Pude Pharmaceutical Co. Ltd; 1640 cell mediums are purchased from Beijing Solarbio Co. Ltd.; fetal calf serum is purchased from American Hyclone Company. Other reagents are analytically pure.

\subsection{Experimental method}

The preparation of nano-micelle through the synthesis of GA-HA and His-HA. FA-HA polymers are synthesized with Wu's method as reference ${ }^{[11]}$. The active ester FA-ES of FA is obtained by FA and the condensation agent DMT-MM in methanol. After rotary evaporating the methanol, FA-ES is slowly added into the ethylene diamine solution, and the intermediate ethylene diamine modified FA-N is stirred at room temperature. The FA-N and hyaluronic acid (HA) after purification by column are put in distilled water. The final product of FA-HA is obtained through DMT-MM as condensation agent. After 5 minutes in ultrasonic fragmentation apparatus, and using 1\% mannitol as the freeze-drying protective agent, nano-micelle lyophilized powders with different degrees of substitution are achieved. They are FA-HA-1, FA-HA-2 and FA-HA-3. His-HA is pre-prepared synthetic hyaluronic acid polymer in the laboratory ${ }^{[7]}$.

Preparation and characterization of FA-HA/His-HA nano-micelle loaded with doxorubicin. Referring to the method proposed by Park et al. ${ }^{[12]}$, FA-HA/His-HA and doxorubicin are dissolved in acetamide and dimethylformamide (containing triethylamine) respectively. Two solutions are mixed slowly and standing still. After dialysis and freeze-drying, the drug loaded nano-micelle (DOX/FA-HA/His-HA) is obtained. Drug loaded nano-micelle (DOX/His-HA) is prepared as control group with His-HA polymer as the carrier.

The drug loaded nano-micelle is prepared as $1 \mathrm{mg} / \mathrm{ml}$ solution; its particle size and Zeta potential are detected by Malvin particle size analyzer. The above solution is dropped to a copper wire mesh coated with support membrane and stained with phosphotungstic acid. The transmission electron microscope is used to observe its form.

Properties of DOX/FA-HA/His-HA nano-micelles. The standard curve is established by ultraviolet spectrophotometer to determine the DOX content in DOX/FA-HA/His-HA solution. Then the drug loading and entrapment efficiency of drug loaded nano-micelles are calculated. Dialysis bag method is used to detect the release amount of drug loaded of nano-micelles at different time and $\mathrm{pH}$ values. In vitro release curve is drawn with time as the abscissa value and cumulative release rate as the ordinate.

Encapsulation efficiency $=\left(\mathrm{W}_{2} / \mathrm{W}_{1}\right) \times 100 \%$

Drug loading capacity $=\left(\mathrm{W}_{2} / \mathrm{W}_{\mathrm{t}}\right) \times 100 \%$

$\mathrm{W}_{1}$ : initial DOX addition; $\mathrm{W}_{2}$ : the amount of DOX detected by nano-micelle solution; $\mathrm{W}_{\mathrm{t}}$ : the total amount of nano-micelle

Cytotoxicity test of DOX/FA-HA/His-HA. HepG2 cells in the period of logarithmic phase are taken out. The cell suspension is adjusted to the concentration of to $3-5 \times 10^{4}$ cells $/ \mathrm{ml}$, and inoculated into 96 hole cell culture plate. After cell adherence, DOX, DOX/His-HA and DOX/FA-HA/His-HA with different drug concentrations are added. After culturing for 48 hours, MTT and DMSO are added in turn. The light absorption values of each hole at $490 \mathrm{~nm}$ are measured by an enzyme labeling instrument to calculate the survival rate of cells.

The survival rate of cells $\left./ \%=\left(\mathrm{A}_{\text {nano drug group }}-\mathrm{A}_{\text {blank }}\right) / \mathrm{A}_{\text {control group }}-\mathrm{A}_{\text {blank }}\right) \times 100 \%$

A nano drug group is the absorbance of cells after DOX/HA-GA treatment; A control group is the absorbance of cells after adding DOX treatment; $\mathrm{A}_{\text {blank }}$ is the absorbance of blank control pores.

Cell uptake test of FA-HA/His-HA nano-micelle. FITC labeled nano-micelles are prepared by fluorescence labeling with FITC. HepG2 cells are seeded into 6 well plates. After cell adherence, FITC-FA-HA/His-HA and DOX/FA-HA/His-HA solutions are added to cultivate for 2 hours respectively. After washing with PBS, the cells are fixed and observed under fluorescence 
microscope.

Detection of antitumor activity of DOX/FA-HA/His-HA nano-micelle. A model of H22 tumor-bearing mice is established. The mice with tumor volume up to $1 \mathrm{~cm}^{3}$ are selected and randomly divided into 4 groups: the negative control group (be injected with equal volume of normal saline), the DOX group, the DOX/His-HA group and the DOX/FA-HA/His-HA group. The DOX dosage of three drug delivery groups is $5 \mathrm{mg} / \mathrm{kg}$; the drug is injected every other day. The mice are weighed at regular intervals. After 14 days of drug administration, the mice are killed; the tumor blocks are taken out to calculate the volume.

Statistical analysis. The statistical analysis is carried out with SPSS 17 statistical software, and the experimental data are expressed as mean \pm standard deviation $(\bar{x} \pm s)$. A single factor analysis of variance is used to test the level of $\alpha=0.05$; the difference $\mathrm{P}<0.05$ means statistical significance.

\section{Experimental Result and Analysis}

\subsection{Properties of DOX/FA-HA/His-HA nano-micelles}

The results show that the DOX/FA-HA/His-HA nano-micelles are well dispersed in a regular sphere (Figure 1). With the increase of FA substitution degree, Zeta potential increases; the particle size of drug loaded nano materials, as well as the drug loading capacity and encapsulation efficiency decrease. This indicates that the modification degree of folic acid affects the physicochemical properties of nano micelles (shown in Table 1). The FA-HA-3/His-HA with smaller particle size is selected for subsequent experiments.

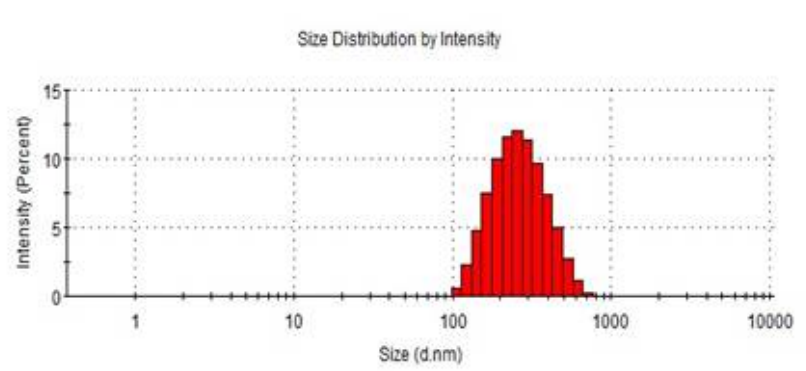

A

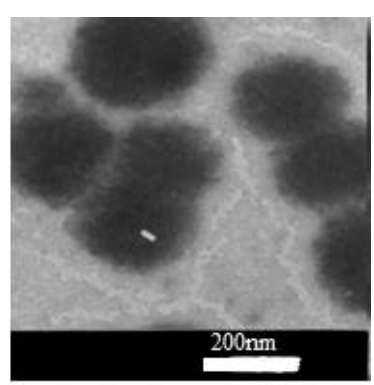

B

Figure1. Size distribution (A) and TEM image (B) of FA-HA/His-HA nano-micelles Table1. Characteristics of DOX/FA-HA/His-HA nano-micelles $(\mathrm{n}=3)$

\begin{tabular}{cccccc}
\hline No. & $\begin{array}{c}\text { drug loading c } \\
\text { apacity }\end{array}$ & $\begin{array}{c}\text { encapsulation } \\
\text { efficiency }\end{array}$ & $\begin{array}{c}\text { partical size } \\
(\mathrm{nm})\end{array}$ & $\begin{array}{c}\text { Polydispersity } \\
\text { index }\end{array}$ & $\begin{array}{c}\text { Zeta point } \\
\text { location }(\mathrm{mV})\end{array}$ \\
\hline FA-HA-1/His-HA & $9.2 \pm 0.4$ & $91.42 \pm 0.9$ & $312.7 \pm 21.4$ & 0.179 & $-12.5 \pm 2.2$ \\
FA-HA-2/His-HA & $9.1 \pm 0.6$ & $90.51 \pm 0.7$ & $286.2 \pm 18.5$ & 0.137 & $-10.4 \pm 1.8$ \\
FA-HA-3/His-HA & $8.9 \pm 0.3$ & $89.72 \pm 1.1$ & $238.4 \pm 14.2$ & 0.117 & $-9.8 \pm 1.3$ \\
\hline
\end{tabular}

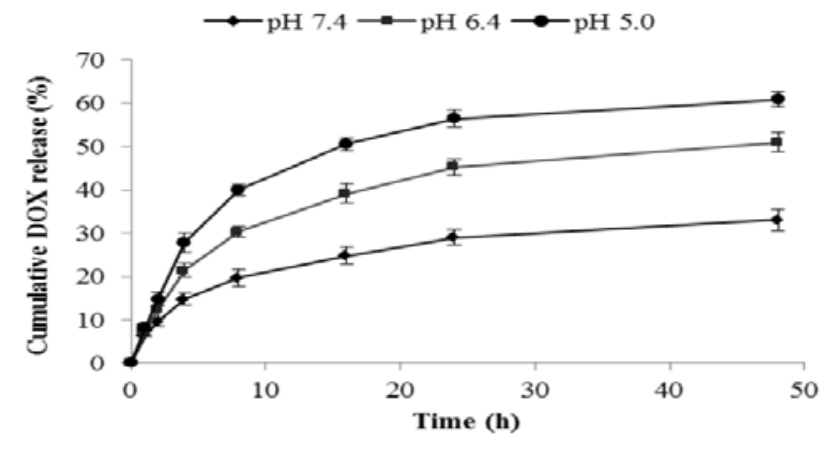

Figure 2. Curve of in vitro DOX release of DOX/FA-HA/His-HA nano-micelles at different pH values 


\subsection{In vitro release of DOX/FA-HA/His-HA nano-micelle}

In this paper, the release rule of drug loaded nano micelles in vitro is studied with FA-HA-3/His-HA as the material. The experiment shows that at different $\mathrm{pH}$ values, the DOX/FA-HA/His-HA nano-micelles have an initial burst effect and then slow release (Figure 2). After 24 hours, the amount of DOX release increases significantly in acidic environment. Among them, the largest amount of DOX released in medium with the $\mathrm{PH}$ value of 5.0, which is 1.98 times of release amount in medium with $\mathrm{pH}$ value of 7.4, indicating that the drug loaded nano micelles are pH-sensitive.

\subsection{Cytotoxicity of nano-micelle DOX/FA-HA/His-HA}

The results of MTT detection show that the proliferation of HepG2 cells is inhibited after three treatments of DOX, DOX/His-HA and DOX/FA-HA/His-HA. The inhibition effects increase with drug concentration. Compared with DOX/His-HA, DOX/FA-HA/His-HA is more effective in anti-tumor activities, and can reach the cell inhibition level of group DOX. This shows that the modification of FA ligands improves the cell intake of nano-micelles and improves the cytotoxicity.

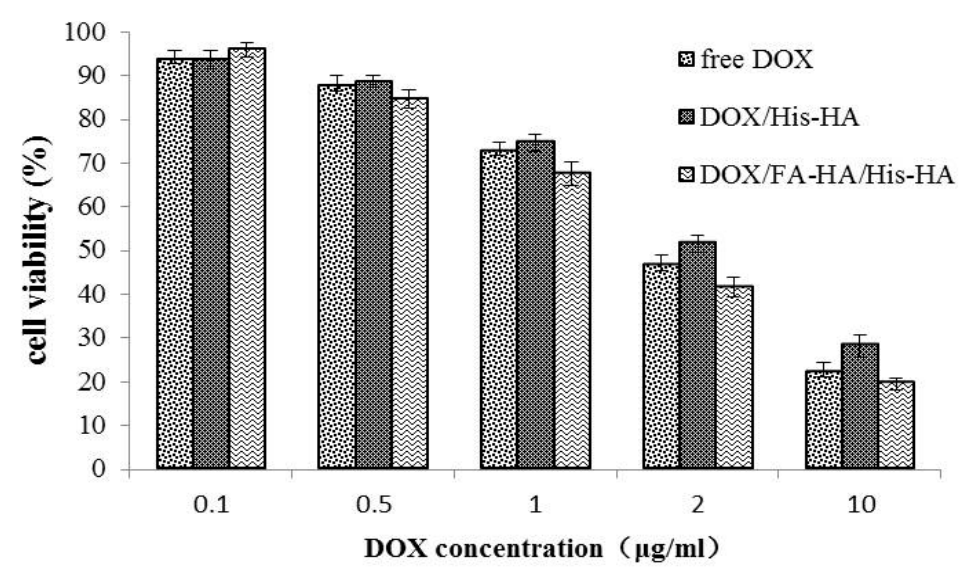

Figure 3. HepG2 cell viability after treatment with DOX, DOX/His-HA and DOX/FA-HA/His-HA nano-micelles
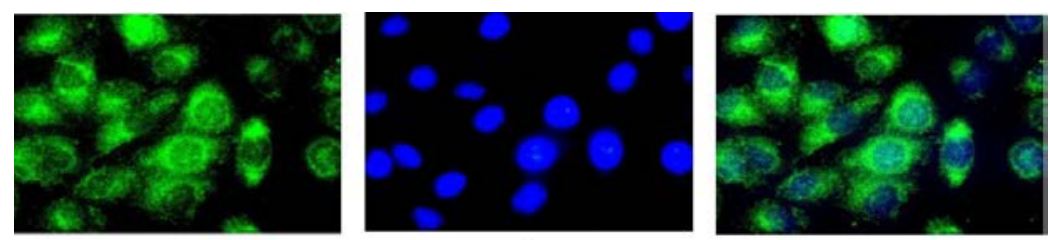

A
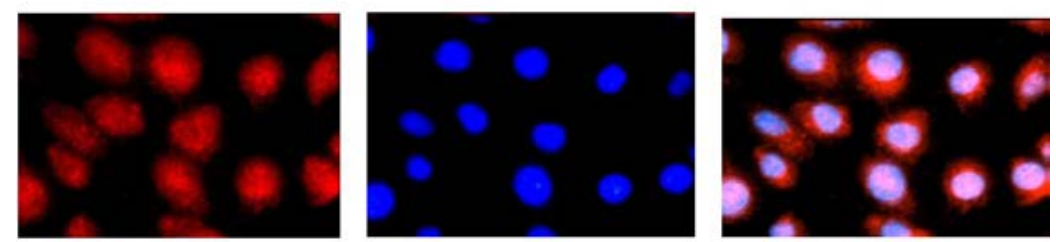

B

Figure 4. Images of cellular uptake of FA-HA/His-HA nano-micelles

FITC: green fluorescence; DOX: red fluorescence; DAPI: blue fluorescence

\subsection{Cellular uptake test of FA-HA/His-HA nano-micelle}

Fluorescence microscope observation shows that after culture of 2 hours, the cytoplasm of HepG2 show green fluorescence (Figure. 4 A), which indicates that FA-HA/His-HA micelles are absorbed by HepG2 cells. In Figure. 4 B, the cytoplasm and nucleus show strong red fluorescence in cytoplasm and nucleus, indicating that DOX has been widely dispersed in cytoplasm and nucleus. 


\subsection{Antitumor activity of DOX/FA-HA/His-HA nano-micelle}

Tumor inhibition test results show that (Figure. 6), after administration, the body weight of DOX group is significantly lower than that of the negative control group (saline) $(\mathrm{P}<0.05)$, and the body weight of DOX/His-HA and DOX/FA-HA/His-HA group is not significantly different from that of the negative control group $(\mathrm{P}>0.05)$. After continuous administration, compared with the negative control group, the tumor growth of the three drug groups is inhibited the. The DOX/FA-HA/His-HA treatment group has the smallest tumor diameter, showing the strongest anti-tumor effect.

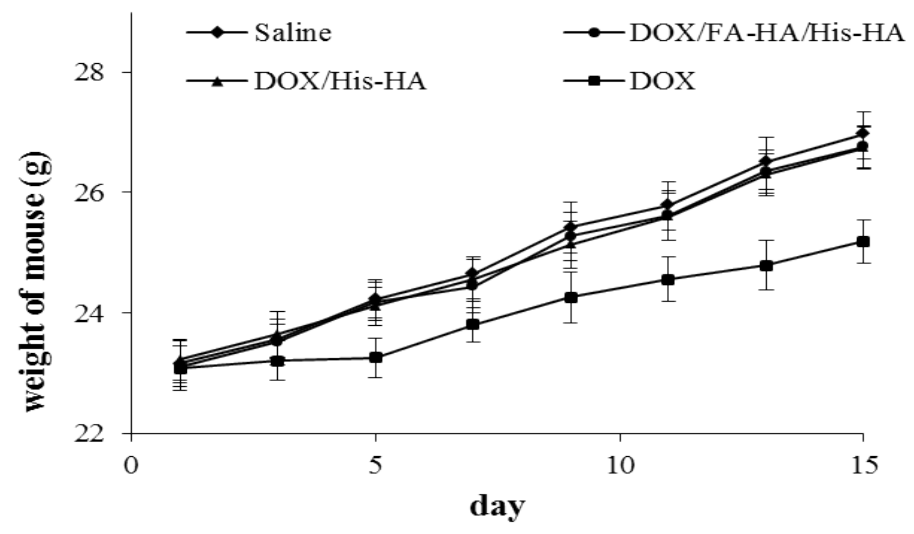

Figure 5. Change of body weight after drug administration

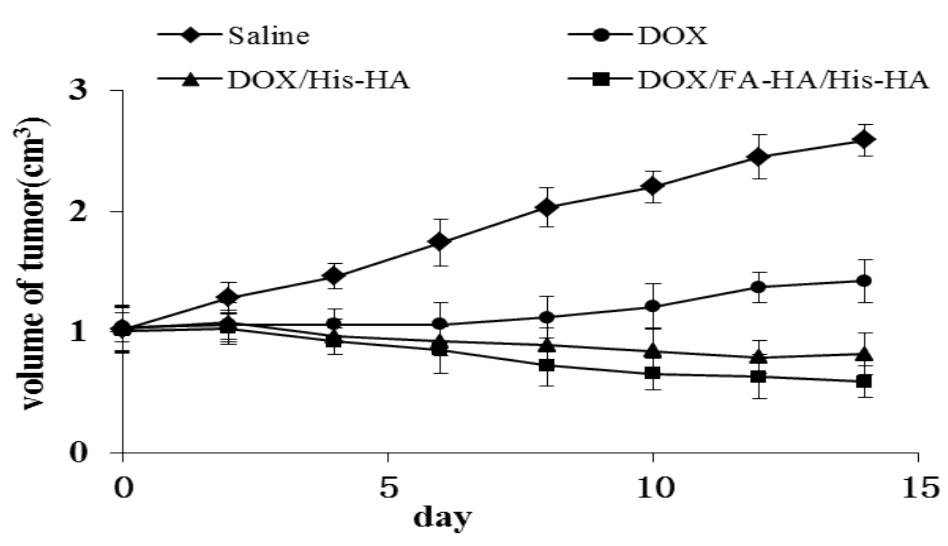

Figure 6. Change of tumor volume after drug administration

\section{Discussion}

In this paper, FA-HA/His-HA mixed nano-micelles are prepared by using folic acid (HA) as the modified group. Using DOX as the model drug, the FA-HA/His-H nano micelles containing DOX are prepared through ultrasonic dispersion method. The particle size of drug loaded nano micelles decreases with the increase of FA substitution degree. This is because the modification of GA increases the hydrophobic core of micelles, making the micelle structure smaller and more compact. In vitro release experiments show that drug releases faster with the decrease of $\mathrm{pH}$ value. This is because in acidic environment, protonation occurs in the imidazole group of His, resulting in lower hydrophobic and stronger hydrophily, which decreases the hydrophobic forces between FA-HA/His-HA micelles. The hydrophobic kernels of nano micelles become loose; embedded DOX is released in large amount ${ }^{[13]}$.

MTT experiments show that the drug loaded nano micelles could effectively inhibit the proliferation of HepG2 cells. Compared with DOX/His-HA, DOX/FA-HA/His-HA has higher cytotoxicity. A possible reason is that, FA-HA/His-HA micelles are ingested by receptor-mediated cells through FA and CD44 receptors, reducing the efflux function of P-glycoprotein on cell membrane and improving the pesticide effects ${ }^{[14]}$.

The anti-tumor experiments show that, compared with the control group, the body weight growth 
of mice in DOX treated group is significantly slower, while the growth rates of two nano-micelle groups do not show significant difference. Since the nano groups can achieve target drug delivery, the toxic and side effects of DOX can be reduced. In addition, compared with the DOX treatment group, DOX/FA-HA/His-HA nano-micelles have stronger tumor suppressor effects. This is because FA-HA/His-HA has targeting ability in the treatment of liver cancer. It can improve the drug concentration in tumor tissues, improving the bioavailability of drugs, and further increasing the tumor inhibition efficiency ${ }^{[15]}$.

\section{Acknowledgement}

Fund Project: This paper is supported by the Foundation for Science and Technology Planning Projects of Colleges and Universities in Shandong (Project No. J17KA141) and Funds for Medical and Health Planning Program of Shandong Province (Project No. 2016WS0673). It is also supported by the Foundation for Science and Technology Projects on the Development of Chinese Medicine in Shandong (Project No. 2015-232, 2017-212) and Funds for Science and Technology Development Projects of Weifang (Project No. 2017YX065, 2016YX011).

\section{References}

[1] Y. Wang, H. Du, G. Zhai, Recent advances in active hepatic targeting drug delivery system, J. Curr Drug Targets. 15 (2014).

[2] M. Akyuz, P. Yazici, H. Yigitbas, et al, Oncologic results of laparoscopic liver resection for malignant liver tumors, J. J Surg Oncol. 113(2016).

[3] S. Naahidi, M. Jafari, F. Edalat, et al, Biocompatibility of engineered nanoparticles for drug delivery. J. J Control Release. 166 (2013).

[4] T. Wei, J. Liu, H. Ma, et al, Functionalized nanoscale micelles improve drug delivery for cancer therapy in vitro and in vivo, J. Nano Lett. 13 (2013).

[5] M. Han, Q. Lv, X.J. Tang, et al, overcoming drug resistance of MCF-7/ADR cells by altering intracellular distribution of doxorubicin via MVP knockdown with a novel siRNA polyamidoamine-hyaluronic acid complex, J. J Control Release. 163(2012).

[6] Y. Zhong, J. Zhang, R. Cheng, et al, reversibly crosslinked hyaluronic acid nanoparticles for active targeting and intelligent delivery of doxorubicin to drug resistant CD44 human breast tumor xenografts, J. J Control Release. 205 (2015).

[7] J.L. Wu, C.G. Liu, X.L. Wang, et al, Preparation and characterization of nanoparticles based on histidine-hyaluronic acid conjugates as doxorubicin carriers, J. J Mater Sci Mater Med. 23 (2012).

[8] F. Dosio, S. Arpicco, B. Stella, E. Fattal, Hyaluronic acid for anticancer drug and nucleic acid delivery, J. Advanced Drug Delivery Reviews. 97 (2016).

[9] K.Y. Choi, H.Y. Yoon, J.H. Kim, S.M. Bae, R.W. Park, Y.M. Kang, et al, Smart nanocarrier based on PEGylated hyaluronic acid for cancer therapy, J. ACS nano. 11 (2011).

[10] L. Zhang, S. Gao, F. Zhang, K. Yang, Q. Ma, L. Zhu, Activatable hyaluronic acid nanoparticle as a theranostic agent for optical/photoacoustic image-guided photothermal therapy, J. ACS nano. 8(2014).

[11] J.L. Wu, G.X. Tian, W.J. Yu, et al, pH-Responsive Hyaluronic Acid-Based Mixed Micelles for the Hepatoma-Targeting Delivery of doxorubicin, J. INT J MOL SCI. 17 (2016).

[12] K. Park, G.Y. Lee, Y.S. Kim, et al, Heparin-deoxycholic acid chemical conjugate as an anticancer drug carrier and its antitumor activity, J. J Control Release. 114 (2006).

[13] L. Qiu, Z. Li, M. Qiao, M. Long, M. Wang, X. Zhang, et al. Self-assembled pH-responsive hyaluronic acid-g-poly((L)-histidine) copolymer micelles for targeted intracellular delivery of 
doxorubicin, J. Acta biomaterialia. 10(2014).

[14] T. Kobayashi, T. Ishida, Y. Okada, et al, Effect of transferrin receptor-targeted liposomal doxorubicin in P-glycoprotein-mediated drug resistant tumor cells, J. Int J Pharm. 329 (2007).

[15] B. Ma, L. He, Y. You, J. Mo, T. Chen, Controlled synthesis and size effects of multifunctional mesoporous silica nanosystem for precise cancer therapy. J. Drug delivery. 25 (2018). 